\title{
Intervertebral infection due to Candida albicans in an intravenous heroin abuser
}

\author{
I F ROWE, ' E D WRIGHT, ${ }^{2}$ C S HIGGENS, ${ }^{1}$ AND J P BURNIE
}

From the Departments of 'Rheumatology and 'Microbiology, Queen Mary's Hospital, Roehampton, London; and the ${ }^{3}$ Department of Medical Microbiology, St Bartholomew's Hospital. West Smithfield, London

SUMMARY A 25 year old woman who had received intravenous heroin over one year previously developed an intervertebral abscess due to infection with Candida albicans. Immunological investigation of this patient showed no evidence of a specific defect in the host response to candida.

Bone and joint infection due to Candida albicans, sometimes in association with other features of disseminated candidiasis, are being diagnosed increasingly, especially in immunocompromised and postoperative patients. ${ }^{1}$ In addition, it has recently been recognised that a characteristic form of systemic candidiasis occurs in heroin addicts. ${ }^{2-4}$ Typically the syndrome comprises folliculitis, particularly of the scalp and beard area, endophthalmitis, and bone or joint lesions, most commonly costochondritis. We report here a patient who developed an intervertebral candidal infection presenting apparently over a year after injection of heroin. It is uncertain why this infection should have developed; we have therefore investigated in some detail the immunological responses of our patient to $C$ albicans.

\section{Case report}

A 25 year old unemployed woman presented with a two month history of progressive central and left sided low back pain. This had started suddenly while on holiday in Greece. Pain was exacerbated by movement, woke her at night up to three hourly, and radiated into the left buttock. Symptoms had not been relieved by non-steroidal anti-inflammatory agents, dihydrocodeine, or physiotherapy. She had otherwise been well with no fever or lymphadeno-

Accepted for publication 4 December 1987

Correspondence to Dr I F Rowe. Department of Rheumatology. Westminster Hospital. London. SWIP 2AP. pathy, but admitted to eating local dairy produce while in Greece. Nine months before presentation she had attended a drug dependency unit for buprenorphine and lorazepam abuse and over one year previously had apparently received heroin intravenously, on one or at most two occasions, without her consent at a party.

On examination she was apyrexial. Abnormal findings were confined to the musculoskeletal system; there was a scoliosis of the lumbar spine concave to the left with loss of normal lordosis and restriction of all movements because of pain. The left hip was held in $10^{\circ}$ of flexion; there were no abnormal neurological signs in the legs.

Investigations showed haemoglobin $99 \mathrm{~g} / 1$; white blood cells (WBC) $18.2 \times 10^{9} / 1$ ( $72 \%$ neutrophils); erythrocyte sedimentation rate (ESR) $112 \mathrm{~mm} / \mathrm{h}$; C reactive protein (CRP) $118 \mathrm{mg} / \mathrm{l}$; alkaline phosphatase $195 \mathrm{IU} / \mathrm{l}$ (normal range (NR) 30-155); $\gamma$-glutamyltransferase 196 IU/l (NR 8-35); bilirubin and aspartate transaminase normal. Radiographs of the lumbar spine showed scoliosis concave to the left 0 with reduction of the L2-3 disc space. There was destruction of the end plates of L2 and L3 with bone $\stackrel{\infty}{-}$ erosion and new bone formation with bridging 0 between these vertebrae (Fig. 1). A few millilitres of grey/green pus aspirated from the lesion through a needle inserted under CT control contained neu- $\mathbb{D}$ trophils but no evidence of bacteria, including acid fast bacilli. C albicans was present on staining, and a pure growth of $C$ albicans was obtained on culture, 


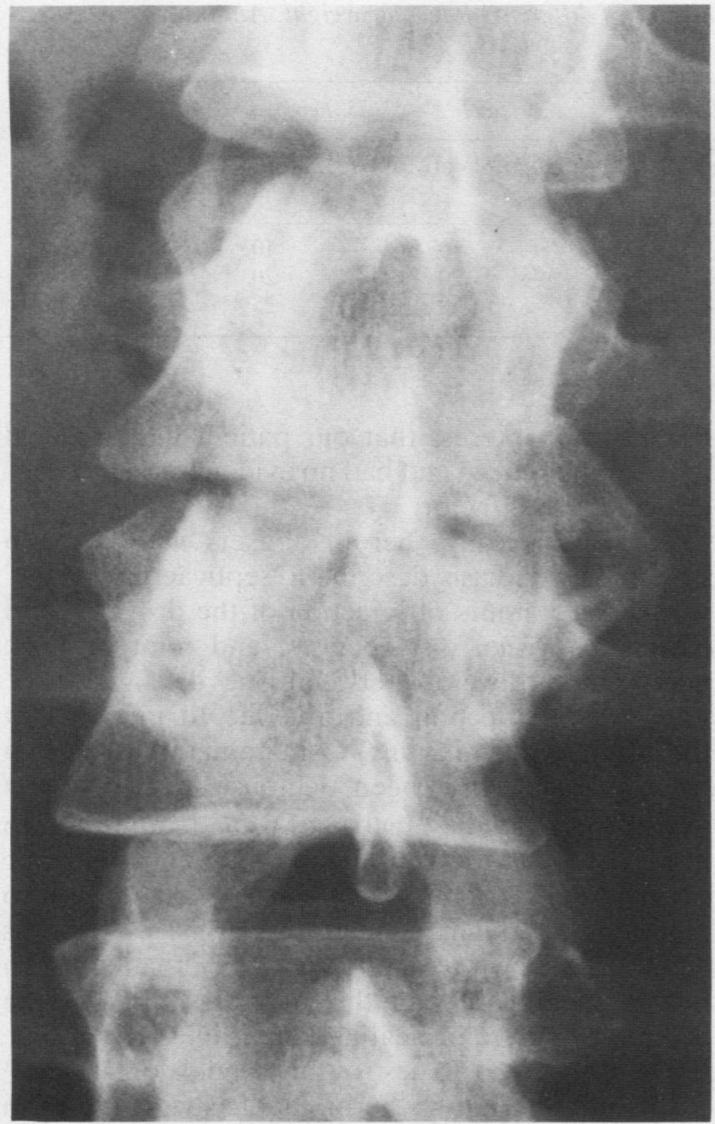

Fig. 1a

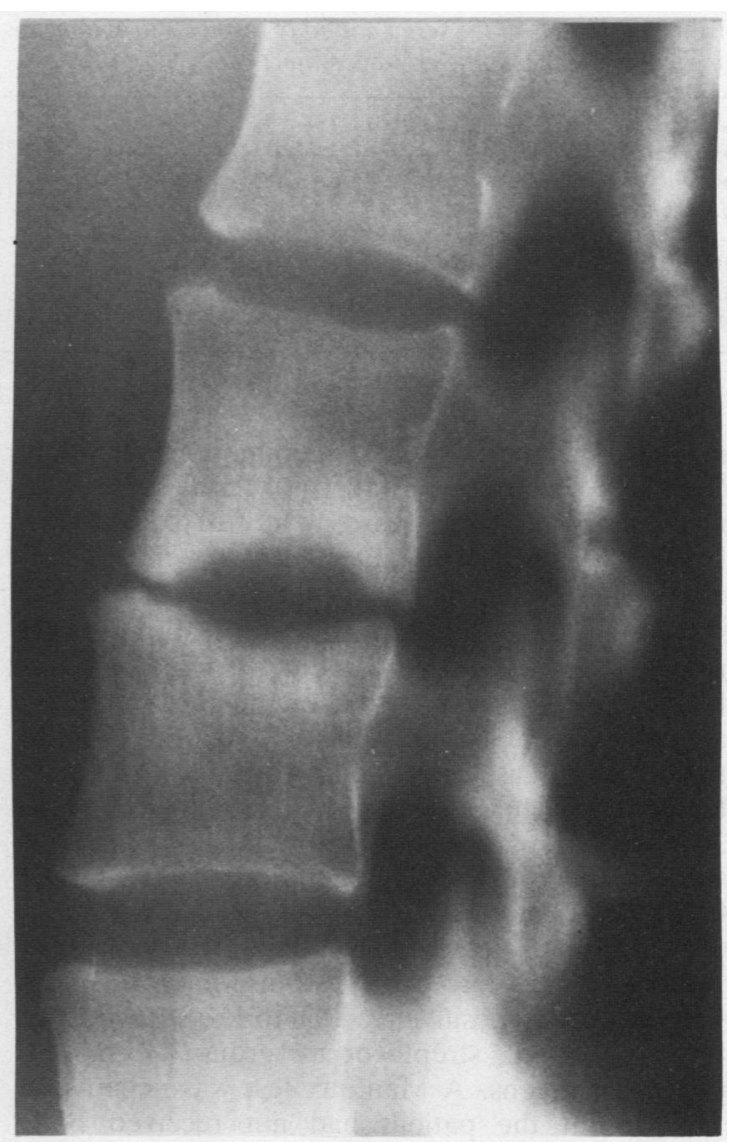

Fig. 1b

Fig. 1 Radiography of the lumbar spine: (a) anteroposterior and (b) lateral views showing reduction of the L2-3 disc space, erosion of the end plates of $L 2$ and $L 3$, and new bone formation with bridging between these vertebrae.

later found to be sensitive to 5-flucytosine, amphotericin B, and ketoconazole. A bone scan showed increased uptake at L2 -3 only and abdominal ultrasound revealed no evidence of psoas abscess. The following serological results were obtained: hepatitis B surface antigen negative; human immunodeficiency virus antibody negative; antistaphylolysins and brucella agglutinins not raised; $C$ albicans precipitins and agglutinins raised (see below).

She was treated with bed rest, transcutaneous nerve stimulation, and naproxen. A central venous (Hickman) line was inserted and she was given intravenous amphotericin $(0.3 \mathrm{mg} / \mathrm{kg} /$ day $)$ and oral flucytosine $35 \mathrm{mg} / \mathrm{kg} / \mathrm{day}$, increasing to $105 \mathrm{mg} / \mathrm{kg} /$ day on the basis of drug levels for two months. Clinical improvement occurred over this period with reduction of back pain, loss of scoliosis, and weight gain: WBC, ESR, CRP, and alkaline phosphatase values returned to normal. Treatment was then started with oral ketoconazole, which was continued for six months.

The patient's immunological responses to $C$ albicans have been studied in some detail at various stages of the illness. $C$ albicans precipitins and agglutinins at presentation were $1 / 4$ (raised $>1 / 1$ ) and $1 / 64$ (raised $>1 / 8$ ) respectively, falling to $1 / 2$ and $1 / 4$ respectively over seven months. A recently developed immunoblotting technique was used to examine the antibody response to individual candidal antigenic bands. ${ }^{5}$ Briefly, $C$ albicans NCTC 3153 was grown on Sabouraud's agar, harvested, fragmented in an Xpress, centrifuged, and the supernatant run in a $10 \%$ sodium dodecyl sulphatepolyacrylamide gel electrophoresis. It was transferred onto nitrocellulose membrane and developed by a 
Table 1 Details of the serial antibody response to $\mathrm{C}$ albicans antigens of different molecular weight. Antibody is measuredent. as height of densitometer trace ( $\mathrm{mm}$ )

\begin{tabular}{|c|c|c|c|c|c|c|c|c|c|c|c|c|c|c|c|}
\hline \multirow{2}{*}{$\begin{array}{l}\text { Apparent } \\
\text { molecular } \\
\text { weight } \\
\text { (kilodaltons) }\end{array}$} & \multicolumn{2}{|c|}{17 Nov } & \multicolumn{2}{|c|}{$13 \mathrm{Dec}$} & \multicolumn{3}{|c|}{$13 \mathrm{Jan}$} & \multicolumn{2}{|c|}{$21 \mathrm{Jan}$} & \multicolumn{2}{|c|}{$29 \mathrm{Jan}$} & \multicolumn{2}{|c|}{$5 \mathrm{Feb}$} & \multicolumn{2}{|c|}{$12 \mathrm{Feb}$} \\
\hline & $\lg M$ & $\operatorname{Ig} G$ & $\lg M$ & $\lg G$ & $\lg M$ & $\lg G$ & & $\lg M$ & $\operatorname{Ig} G$ & $\lg M$ & $\operatorname{Ig} G$ & $\lg M$ & $\lg G$ & $\lg M$ & $\lg G$ \\
\hline 92 & - & 10 & - & 10 & - & 10 & & - & 15 & - & 10 & - & 10 & - & 10 \\
\hline 60 & - & 15 & - & 15 & - & 15 & & - & 25 & - & 20 & - & 20 & - & 20 \\
\hline 47 & 10 & 10 & - & 10 & - & 20 & 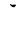 & - & 30 & - & 20 & - & 25 & - & 25 \\
\hline
\end{tabular}

modified enzyme linked immunosorbent assay (ELISA) technique. Both IgM and IgG were measured and the response quantified by a JoyceLoebl scanning densitometer as described in detail elsewhere. ${ }^{5}$ The earliest serum (at presentation) showed a small (densitometer height $10 \mathrm{~mm}$ ) IgM response to an antigenic band of 47 kilodaltons (Table 1). This serum also contained IgG antibody against bands of 47, 60, and 92 kilodaltons. Resolution of the disease correlated with an increase in the level of IgG against the 47 kilodalton band to $30 \mathrm{~mm}$. The patient's total immunoglobulin levels were normal; C3 (at presentation) $1.66 \mathrm{~g} / \mathrm{l}$ (NR $0 \cdot 7-1 \cdot 6)$; $\mathrm{C} 4$ normal.

Skin tests to detect delayed-type hypersensitivity reactions (Multitest cmi, Institut Merieux, Lyon, France) showed positive reaction to Proteus mirabilis, tetanus toxoid, streptococcus group $\mathrm{C}$, and $C$ albicans antigens. A Mantoux test was negative at $1 / 1000$, but the patient had not received BCG vaccination nor suffered from tuberculosis. Studies of T lymphocyte subsets (FACS analyser, Becton Dickinson) showed some activation of $T$ cells and some $\mathrm{T}$ cell recruitment: total lymphocytes $2.6 \times 10^{9} / \mathrm{l}\left(\mathrm{NR} 1.2-3.5 \times 10^{9} / \mathrm{l}\right)$; total T cells, $80 \%$ (NR 51-78); D+T cells (activated) $11 \%$ (normally infrequent); Leu 2+3- (suppressor) 38\% (NR 18-35); Leu 3+2- (helper) $27 \%$ (NR 27-49); Leu $2+3+$ (common thymocytes) $9 \%$ (NR 1-8).

Tests of the ability of the patient's neutrophils to phagocytose and kill $C$ albicans intracellularly," performed six months after initiation of treatment, were normal.

\section{Discussion}

In our patient the site of the abscess in the spine implies the haematogenous origin of the infecting organism, $C$ albicans. Several risk factors for candidaemia have been recognised, including host immune compromise, gastrointestinal surgery, burns, and trauma.' Candidaemia may also occur iatrogenically after intravenous or arterial catheterisation, or may occur after intravenous drug abuse. It is therefore of interest that our patient had receive $\frac{0}{5}$ intravenous heroin and had no evidence of any othei identified risk factor.

Characteristically, heroin abusers who develoris systemic candidiasis describe a 'septicaemic' phase ${ }^{N}$ within a few hours of injection of the drug. Painful folliculitis, visual disturbances, and joint or back pain usually develop between one day and three weeks after injection and are due to infection a® these sites by $C$ albicans. Contamination of heroino by candida has not been demonstrated; infectiorso may result from the practice of using lemon juice to dissolve certain forms of the drug, ${ }^{7}$ and an epidemis of candidal endophthalmitis has been traced to several contaminated plastic lemons. ${ }^{89}$ Lemon\& have been shown to support growth of candida; a unexplained feature of this hypothesis is that the common practice of heating lemon juice to dissolve the heroin is likely to destroy any candida organisms.

It is not unusual for heroin addicts with this forn of systemic candidiasis to present with only one of two of the typical triad of clinical features. Infectio of the vertebral column has been reported io association with this syndrome, and drug abuserș may develop features of infection after only ons heroin injection. Although our patient had candide infection involving bone and a history of heroig abuse, she had apparently not had the 'septicaemic's phase of illness typically associated with this syno drome, nor did she have any evidence of cutaneous or ocular involvement. If the history is reliable from? our patient, the notable feature which appears toु distinguish her case from those with this syndrome is the long time interval of over a year between drug injection and the development of symptoms. Thi raises the possibility that in some individuals infec tion may be subclinical for a longer period of time of that in her case heroin injection was not relevant too the subsequent candidal infection.

Although various immunological abnormalitie have been reported in chronic heroin addicts, ${ }^{10}$ ' 10 the reason why our patient or those who supposedly inject candida intravenously with heroin should develop candidal infection of bone is not clear. WeO 
therefore, sought to investigate our patient's immunological responses to $C$ albicans in some detail. There appeared to be no abnormality of the humoral antibody response to $C$ albicans antigens. The pattern of IgG antibody response on immunoblot analysis was unremarkable and was a pattern often seen in patients with systemic candidiasis. Of interest was the IgG antibody response to a 47 kilodalton antigen; this has previously been seen in patients who have recovered from systemic candidiasis and is therefore of good prognostic significance. $^{512}$ Similarly, no abnormality of delayed-type hypersensitivity to $C$ albicans antigens was detected. Processes of phagocytosis and intracellular killing are thought to be of importance in providing resistance to candidal infection ${ }^{1}$; no abnormality of these functions could be demonstrated in our patient's neutrophils.

Good responses to treatment for systemic candidiasis in heroin abusers have generally been obtained; the best results for bone, joint, and ocular infection appear to have been with amphotericin B and flucytosine. ${ }^{2}$ Similarly, our patient showed a good response to this treatment. Surgical treatment of bone and joint lesions has appeared, in general, not to be necessary.

With the growing problem of drug abuse it is likely that systemic candidiasis will be seen increasingly in British practice and, as bone and joint lesions may occur without other features of infection, the diagnosis should be considered in heroin abusers presenting with musculoskeletal symptoms to rheumatologists and orthopaedic surgeons. Our patient raises the possibility that candidal infection of bone may occur a considerable time after heroin injection or that sporadic cases of infection may arise without known risk factors.

We thank Dr R J Hay, Institute of Dermatology. London. for advice regarding management of this patient: Professor D W R Mackenzie. Mycological Reference Laboratory. Central Public Health Laboratory. Colindale. for measuring $C$ albicans agglutinins and precipitins. determining antifungal sensitivities. and monitoring blood levels of flucytosine: Dr N Byrom. Westminster Hospital. for measuring T cell subsets: Dr A J Pinching. St Mary's Hospital Medical School. for performing the phagocytosis studies: and Miss A M Brown for expert secretarial assistance.

\section{References}

1 Edwards J E. Candida species. In: Mandell G L. Gordon Douglas R, Bennett J E, eds. Principles and practice of infectious diseases. New York: Wiley, 1985: 1435-47.

2 Collignon P J, Sorrell T C. Disseminated candidiasis. Evidence of a distinctive syndrome in heroin abusers. $\mathrm{Br}$ Med J 1983; 287: 861-2.

3 Dupont B. Drouet E. Cutancous, ocular, and osteoarticular candidiasis in heroin addicts: new clinical and therapeutic aspects in 38 patients. $J$ Infect Dis 1985: 152: 577-90.

4 Hay R J. Systemic candidiasis in heroin addicts [Editorial]. $\mathrm{Br}$ Med J 1986; 292: 1096.

5 Matthews R C. Burnic J P. Tabaqchali S. Immunoblot analysis of the serological response in systemic candidosis. Lancet 1984; ii: $1415-8$.

6 Bridges C G. Dasilva G L. Yamamura M. Valdimarsson H. A radiometric assay for the combined measurement of phagocytosis and intracellular killing of Candida albicans. Clin Exp Immunol 1980: 42: 226-33.

7 Newton-John H F. Wise K. Looke D F M. The role of lemon juice in disseminated candidiasis of heroin abusers. Med J Aust 1983: 140: 780-1.

8 Servant J B, Dutton G N, Ong-tone L, Barrie T, Davey C. Candidal endophthalmitis in Glaswegian heroin addicts: report of an epidemic. Trans Ophthalmol Soc UK 1985: 104: 297-308.

9 Shankland G S. Richardson M D, Dutton G N. Source of infection in Candida endophthalmitis in drug addicts. $\mathrm{Br} \mathrm{Med} \mathrm{J}$ 1986: 292: 1106-7.

10 Brown S M. Stimmel B. Taub R N. Kochwa S, Rosenficld R E. Immunologic dysfunction in heroin addicts. Arch Intern Med 1974: 134: 1001-6.

11 McDonough R J, Madden J J, Falek A. et al. Alteration of T and null lymphocyte frequencies in the peripheral blood of human opiate addicts: in vivo evidence for opiate receptor sites on T lymphocytes. J Immunol 1980; 125: 2539-43.

12 Strockbine N A. Largen M T, Zweibel S M. Buckley H R. Identification and molecular weight characterization of antigens from Candida albicans that are recognised by human sera. Infect Immun 1984; 43: 715-21. 